\title{
The Influence of the Direction of the Channels of the Gas Swirlers on the Properties of the Flow of the Plasma Jet under Implementation Theoretical Basis and Technical Solutions for Recycling Technogenic Waste
}

\section{Roman Aleksandrovich Okulov ${ }^{1,2}$, Konstantin Ivanovich Sarsadskikh ${ }^{1}$, Sergey Anatolievich Ilinykh ${ }^{1}$, and Mikhail Nikolayevich Zakharov ${ }^{1}$}

${ }^{1}$ Institute of Metallurgy of the Ural Branch of the Russian Academy of Sciences, Ekaterinburg, 620016, Russia

${ }^{2}$ Federal State Autonomous Educational Institution of Higher Education «Ural Federal University named after the first President of Russia B N Yeltsin», 620002, Russia

\section{Abstract}

A variant of the use of plasmatrons for the disposal of industrial waste and the

Corresponding Author:

Roman Aleksandrovich Okulov

okulov.roman@gmail.com

Published: 31 December 2020

Publishing services provided by

Knowledge E

(c) Roman Aleksandrovich

Okulov et al. This article is

distributed under the terms of

the Creative Commons.

Attribution License, which

permits unrestricted use and

redistribution provided that the

original author and source are

credited.

Selection and Peer-review under the responsibility of the TECHNOGEN-2019 Conference Committee. organization of non-waste production is proposed in this article, which presents the design for the functioning equipment. A computer experiment was carried out to determine the parameters of the flow of the plasma jet. The results of a computer experiment are confirmed by a full-scale experiment. Conclusions and results are of practical benefit to developers and consumers of technological equipment.

Keywords: plasmatron, flow, plasma, jet, temperature, velocity

\section{Introduction}

The tasks of finding solutions to the issues of reuse of enterprize waste and increasing waste-free production require solutions at present. In the framework of the proposed scientific work, the prospects of using plasmatrons for tasks on the implementation of the theoretical foundations and technical solutions for the disposal of industrial waste with maximum extraction of components and the organization of non-waste production are considered.

Plasmatrons are widely used in various fields of production [1]. One of the possible ways of using plasmatrons is the operation to remove contaminants from surfaces of various configurations and shapes, including from the surface of the shavings, with its subsequent use as a raw material for the production of powder materials by plasma spraying [2]. To implement this method of using plasmatrons, it is important to study the parameters of the plasma jet (temperature, speed, etc.). In turn, the parameters of the plasma jet depend on the shape of the internal channel of the plasmatron. 
A plasma installation for coating and producing powders is operating at the IMET Ural Branch of the Russian Academy of Sciences. In order to demonstrate the profile of the parts forming the shape of the internal channel of the plasmatron, Figure 1 shows a section along the axis of the head part of the plasmatron, which is part of our installation.

It is of interest to study the effect of the configuration of the gas swirlers on the parameters of the flow of the plasma jet. Gas swirlers are cylindrical parts with a through central hole. 6 channels directed tangentially are brought to the central hole of the gas swirl. Plasma-forming gas is supplied through the channels of the gas swirlers. The purpose of the channels is to create a swirl to form a flow in the form of a spiral and to prevent the burning of parts forming the internal form of the plasmatron and the chamber in which the arc burns. Two options of the directions of the channels of the upper and lower gas swirlers are possible.

Figure 1 shows scheme of the cross section of the head part of the plasmatron. There are two options of the construction of channels of the swirlers depending on the direction of gas swirl: in one direction or in opposite directions.

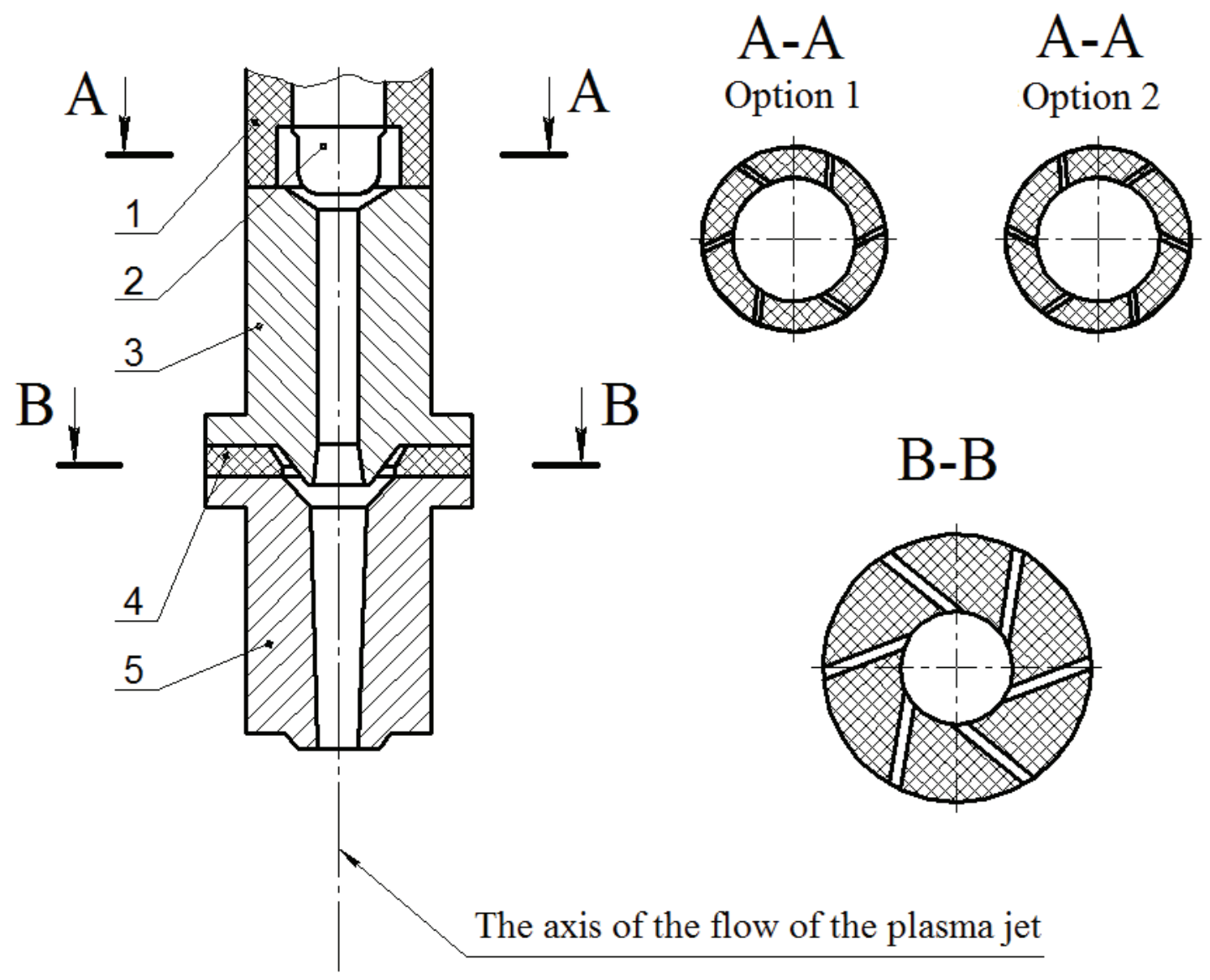

Figure 1: Scheme of the cross section of the head part of the plasmatron 1 - upper gas swirler; 2 - cathode; 3 - interelectrode insert; 4 - lower gas swirler; 5 - anode 


\section{Creating a computer model of the process and conduct- ing a mathematical experiment}

The purpose of the presented scientific work is to perform a comparative analysis of the laws of the distribution of temperature and velocity along the axis of the flow of the plasma jet for two variants of the direction of the channels in swirlers.

SolidWorks software package was used to achieve this goal. The software package allows you to perform a solution by the finite element method [3]. A three-dimensional mathematical model was created that adequately describes laboratory equipment (functioning plasmatron). The values of temperature and flow velocity along the axis of the plasma jet were determined using a computer experiment. The geometric parameters of the model and the type of gas medium are selected in accordance with those used in the existing experimental setup. The initial data used in the simulation by the finite element method are: gas type - argon (introduced through the swirlers), gas volume flow rate - $50 \mathrm{l} / \mathrm{min}$; initial incremental gas pressure - $1.5 \mathrm{~atm}$; the initial plasma temperature is $7000 \mathrm{~K}$. The design of the plasmatron was consisted of the anode with a conical confusor section with a diameter transition from 11 to $8 \mathrm{~mm}$ (from DH to D) and a anode confusor part length $58 \mathrm{~mm}$. The results are presented in Figure 2.

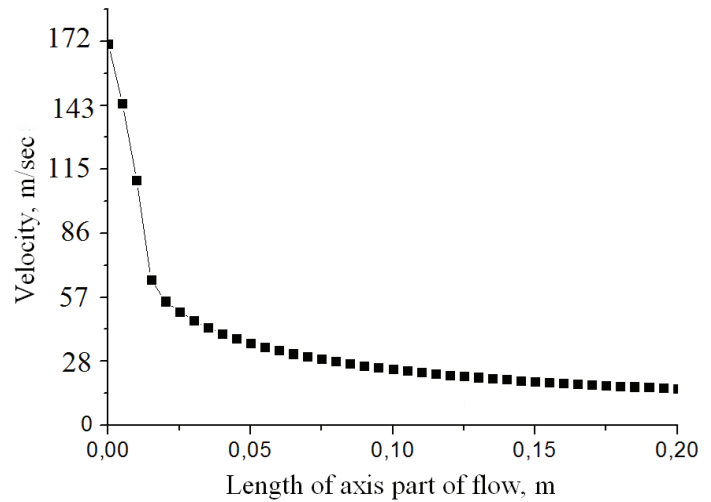

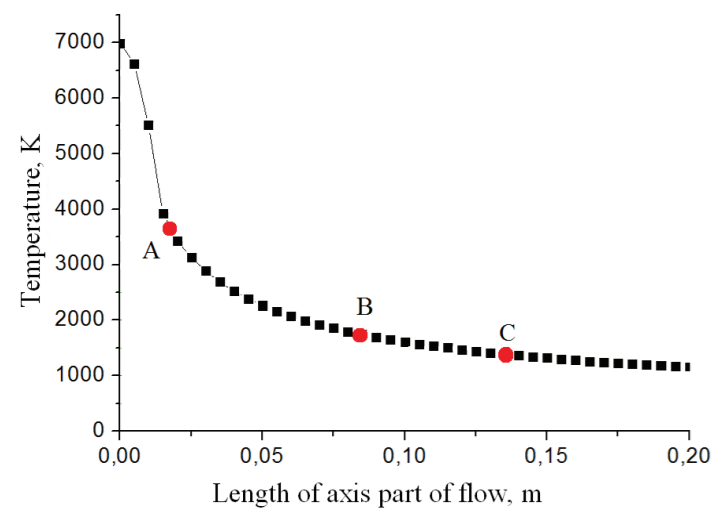

$\mathrm{b}$

Figure 2: Graphs of the distribution of velocity (a) and temperature (b) along the axis of the flow of the plasma jet. According to practical experiment: point A (tungsten), point B (steel), point C (copper).

The software package used allows us to consider the distribution patterns in cross sections of the numerical values of the modeled quantities along the axis of the flow of the plasma jet. Figure 3 presents data on the distribution of the temperature of the flow of the plasma jet. 


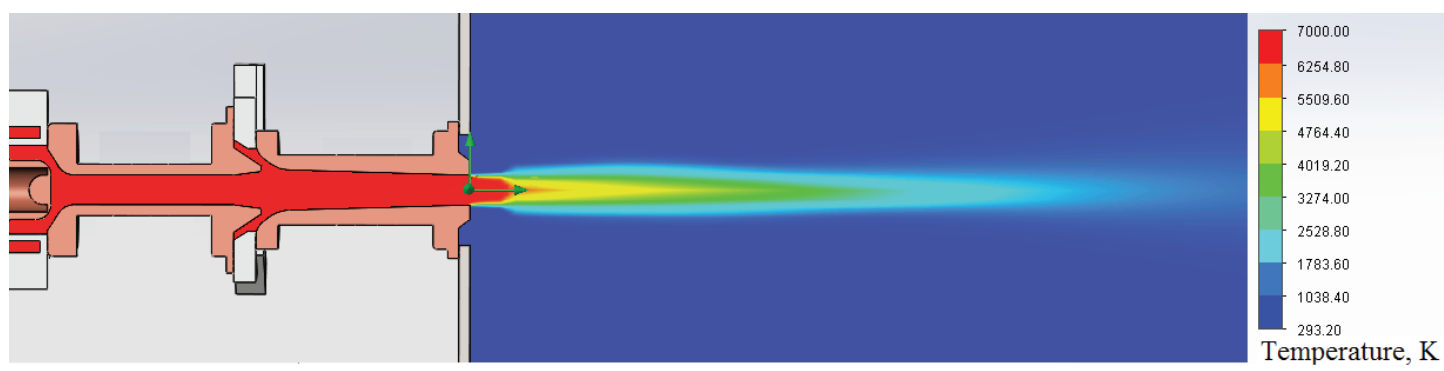

Figure 3: The distribution of the temperature of the flow of the plasma jet.

\section{Description of the full-scale experiment and verification of the theoretical results of a computer experiment}

A full-scale experiment was carried out in order to confirm the adequacy of the computer model. A comparative analysis of the results of determining the temperature of the flow of the plasma jet along its axis at the exit from the plasmatron at three points for theoretical and full-scale experiments is performed. We used rods with a diameter of 3 mm from various materials: copper M1 GOST 859-2014 (melting point 1357 K), Steel 10 GOST 1050-88 (melting point $1758 \mathrm{~K}$ ) and tungsten lanthanized TS 48-19-27-88 (melting point $3653 \mathrm{~K}$ ). The rods were used to determine the temperature of the plasma jet by observing the moment of melting of the rod material. The rods were alternately placed in the manipulator. The manipulator fixed the position of the rod perpendicular to the axis of the flow of the plasma jet so that one end of the rod was on the axis of the flow of the plasma jet and the other was clamped in the manipulator. The manipulator was located at a distance of $0.5 \mathrm{~m}$ from the plasmatron. The manipulator was mixed in space at a speed of $50 \mathrm{~mm} / \mathrm{min}$ in a direction parallel to the axis of the flow of the plasma jet towards the plasmatron. The distance from the free end of the rod to the end plane of the anode (plasmatron exit) was recorded.

The temperature of the surface of the free end of the rod during the melting point was recorded using an optical pyrometer (type EOP-66 No. 240 GOST 5.278). At the moment when the process of melting the rod material began, the distance from the free end of the rod to the plasmatron was recorded. The results are averaged over three parallel experiments with each rod material and compared with the results of a computer experiment (Table 1 and Figure 2). A comparative analysis of the results of full-scale and theoretical experiments showed satisfactory convergence. 
TABLE 1: Distances from the rod to the anode end surface of the plasmatron when the melting temperature on its surface is reached according to the results of theoretical and full-scale experiments

Material
Copper M1
Steel 10
Lantanized tungsten

$\begin{gathered}\text { Theoretical } \\ \text { experiment }(\mathrm{mm})\end{gathered}$
141
82
19

\begin{tabular}{|c|}
\hline $\begin{array}{c}\text { Full-scale } \\
\text { Experiment }(\mathrm{mm})\end{array}$ \\
\hline 135 \\
85 \\
20 \\
\hline
\end{tabular}

\begin{tabular}{|c|}
\hline Measurement Error (\%) \\
\hline 4,4 \\
3,5 \\
5,0 \\
\hline
\end{tabular}

\section{The effect of swirlers on the speed and temperature of the flow of the plasma jet}

Based on the computer model developed and tested for compliance with the real process, we performed a comparative analysis of the temperature and velocity distribution along the axis of the plasma flow for two variants of the direction of the channels in swirlers. In the adopted computer model, the following parameters were used: argon - plasma-forming gas, plasma-forming gas pressure - $0.15 \mathrm{MPa}$; the initial plasma temperature is $7000 \mathrm{~K}$. In order to increase the accuracy of the simulation results, three parallel computer calculations were performed. For the obtained values of the sought quantities, the average values were calculated and the results were presented (Figure 4).

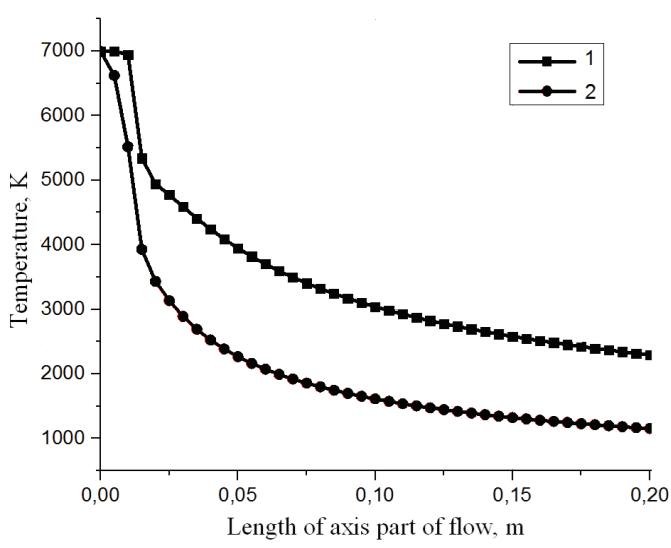

a

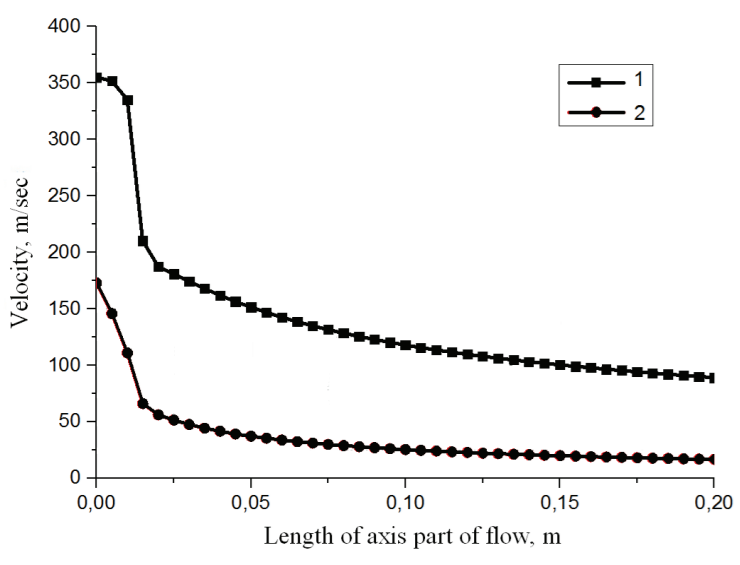

b

Figure 4: Graphs of the distribution of temperature (a) and velocity (b) along the axis of the flow of the plasma jet for two options of the design of channels of the swirlers: 1 - in one direction; 2 - in opposite directions.

\section{Conclusions}

As follows from the data presented, when using swirlers with channels running in one direction, the temperature and velocity of the plasma jet along the axis of the plasma 
flow of the plasmatron are higher than in the opposite case (when the channels are directed towards each other).

As a result of the presented work, it was demonstrated how the choice of the case of the embodiment of channels in swirlers affects the parameters of the plasma flow along its axis. The data presented as part of the work can be used in the development and improvement of process technology and technological equipment for the implementation of solutions for the disposal of industrial waste to achieve maximum component conversion and the organization of non-waste production. The presented results are of practical use in the design of plasmatrons with the aim of predicting the parameters of the plasma jet used in the processing of industrial waste.

The work was carried out according to the state assignment for IMET UB RAS. The reported study was funded by RFBR, project number 20-21-00063 (Rosatom).

\section{References}

[1] Klimenko, A. A. and Lyapin, G. K. (2010). Constructions of Electric Arc Plasma Torches. Moscow: Publishing House of Moscow State Technical University, p. 56.

[2] Anakhov, S. V., Pykin, Y. A. and Matushkin, A. V. (2016). Welding International, issue 30, pp. 408-412.

[3] Matushkin, A. V. (2017). Improving the Gas-vortex Stabilization System of Electric Arc Plasmatrons for Metal Cutting. (PhD dissertation, Ural Federal University named after the first President of Russia B.N. Yeltsin, 2017). 